PROCEEDINGS OF THE

AMERICAN MATHEMATICAL SOCIETY

Volume 127, Number 12, Pages 3685-3695

S 0002-9939(99)05542-2

Article electronically published on August 5, 1999

\title{
MORSE INDICES AND EXACT MULTIPLICITY OF SOLUTIONS TO SEMILINEAR ELLIPTIC PROBLEMS
}

\author{
JUNPING SHI AND JUNPING WANG
}

(Communicated by David S. Tartakoff)

\begin{abstract}
We obtain precise global bifurcation diagrams for both one-sign and sign-changing solutions of a semilinear elliptic equation, for the nonlinearity being asymptotically linear. Our method combines the bifurcation approach and spectral analysis.
\end{abstract}

\section{INTRODUCTION}

Consider the nonlinear semilinear problem:

$$
\begin{cases}\Delta u+\lambda u-f(u)=0 & \text { in } \Omega, \\ u=0 & \text { on } \partial \Omega,\end{cases}
$$

where $\Omega$ is a bounded smooth domain in $\mathbf{R}^{n}, n \geq 1$, and $\lambda$ is a real parameter.

We assume that $f$ satisfies

(f1) $f \in C^{1}(\mathbf{R}, \mathbf{R}), f(0)=0, f^{\prime}(0)>0$;

(f2) $\lim _{|u| \rightarrow \infty} f(u) / u=f^{\prime}(\infty)>0$;

(f3) $f(u) / u$ is increasing in $(0, \infty)$ and is decreasing in $(-\infty, 0)$.

Bifurcation theory implies that global branches of solutions bifurcate from the line of trivial solutions $(\lambda, 0) \subset \mathbf{R} \times C^{2}(\bar{\Omega})$ at simple eigenvalues. But little is known about the whole solution set of (1.1). In this paper, under an eigenvalue separation condition (see (1.5)), we obtain a full description of the first $N$ solution curves which bifurcate from the line of trivial solutions.

To state our results, we first introduce some notation. We denote by $\lambda_{k}$ the $k$-th eigenvalue of

$$
\begin{cases}\Delta \phi+\lambda \phi=0 & \text { in } \Omega, \\ \phi=0 & \text { on } \partial \Omega .\end{cases}
$$

Throughout the paper, we assume that all $\lambda_{k}$ 's are simple. We also define

$$
\lambda_{k}^{0}=\lambda_{k}+f^{\prime}(0), \quad \lambda_{k}^{\infty}=\lambda_{k}+f^{\prime}(\infty), \quad \text { and } \lambda_{k}^{M}=\lambda_{k}+\sup _{u \in \mathbf{R}} f^{\prime}(u) .
$$

By (f3), $f^{\prime}(u) \geq f(u) / u$. Therefore, for $k \in \mathbf{N}$ and $f$ satisfying (f1-3), $\lambda_{k}^{0}<\lambda_{k}^{\infty} \leq$ $\lambda_{k}^{M}$. We define the Morse index $M(u)$ of a solution $u$ to (1.1) to be the number of

Received by the editors February 28, 1998.

1991 Mathematics Subject Classification. Primary 35J25, 35B32; Secondary 35J60, 35P30.

Key words and phrases. Exact multiplicity, bifurcation, eigenvalue comparison.

(C)1999 American Mathematical Society 


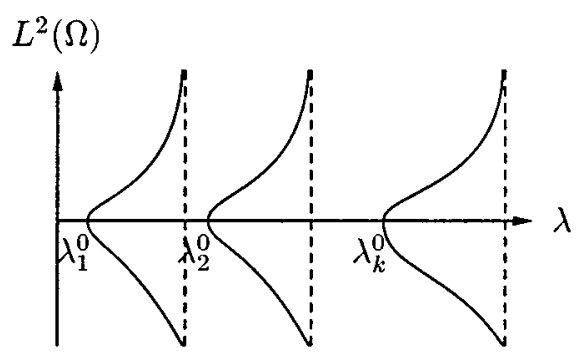

Figure 1. Bifurcation diagram for Theorem 1.1

negative eigenvalues of the following problem:

$$
\begin{cases}\Delta \phi+\lambda \phi-f^{\prime}(u) \phi=-\mu \phi & \text { in } \Omega, \\ \phi=0 & \text { on } \partial \Omega .\end{cases}
$$

If $u$ is a solution to (1.1), and 0 is not an eigenvalue of (1.4), then $u$ is a nondegenerate solution, otherwise it is degenerate. Our first main theorem is:

Theorem 1.1. Suppose that $f$ satisfies (f1), (f2) and (f3), and for $k=1,2, \cdots, N$, we assume that

$$
\lambda_{k}^{M} \leq \lambda_{k+1}^{0} .
$$

Then, for $k=1,2, \cdots, N$ (see Figure 1)

(1) (1.1) has exactly two nontrivial solutions $u_{k}^{+}(\lambda, \cdot)$ and $u_{k}^{-}(\lambda, \cdot)$ for $\lambda \in\left(\lambda_{k}^{0}, \lambda_{k}^{\infty}\right)$, and has only the trivial solution for $\lambda \in\left[\lambda_{k}^{\infty}, \lambda_{k+1}^{0}\right]$.

(2) For $\lambda \in\left(\lambda_{k}^{0}, \lambda_{k}^{\infty}\right)$, all nontrivial solutions of (1.1) lie on two smooth curves $\Sigma_{k}^{ \pm}=\left\{\left(\lambda, u_{k}^{ \pm}(\lambda, \cdot)\right): \lambda \in\left(\lambda_{k}^{0}, \lambda_{k}^{\infty}\right)\right\}, \Sigma_{k}^{+}$and $\Sigma_{k}^{-}$join at $\left(\lambda_{k}^{0}, 0\right)$, and

$$
\lim _{\lambda \rightarrow\left(\lambda_{k}^{\infty}\right)^{-}}\left\|u_{k}^{ \pm}(\lambda, \cdot)\right\|_{L^{2}(\Omega)}=\infty .
$$

(3) For a solution $(\lambda, u) \in \Sigma_{k}^{+} \cup \Sigma_{k}^{-}$, $u$ is nondegenerate and the Morse index $M(u)=k-1$.

Remark. - If in addition to (f1-3), we also assume that $f$ is $C^{2}$ and $u f^{\prime \prime}(u) \geq 0$, then it is easy to see that $\lambda_{k}^{M}=\lambda_{k}^{\infty}$, but in general $\lambda_{k}^{M}>\lambda_{k}^{\infty}$.

- For $(\lambda, u) \in \Sigma_{1}^{ \pm}, u$ is of one sign, so we can assume that for $(\lambda, u) \in \Sigma_{1}^{+}$, $u>0$, and for $(\lambda, u) \in \Sigma_{1}^{-}, u<0$.

If we replace (f3) by

(f4) $f(u) / u$ is decreasing in $(0, \infty)$ and is increasing in $(-\infty, 0)$, then we have a similar result:

Theorem 1.2. Suppose that $f$ satisfies (f1), (f2) and ( $f 4$ ), and for $k=1,2, \cdots, N$, we assume that $\lambda_{k}^{0} \leq \lambda_{k+1}+\inf _{u \in \mathbf{R}} f^{\prime}(u)$. Then, for $k=1,2, \cdots, N-1$ (see Figure 2)

(1) (1.1) has exactly two nontrivial solutions $u_{k}^{+}(\lambda, \cdot)$ and $u_{k}^{-}(\lambda, \cdot)$ for $\lambda \in\left(\lambda_{k}^{\infty}, \lambda_{k}^{0}\right)$, and has only the trivial solution for $\lambda \in\left[\lambda_{k}^{0}, \lambda_{k+1}^{\infty}\right]$. 


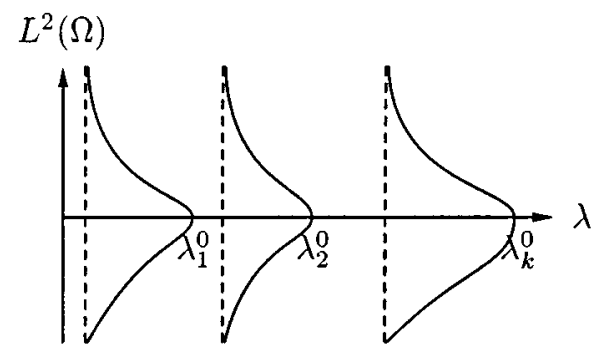

Figure 2. Bifurcation diagram for Theorem 1.2

(2) For $\lambda \in\left(\lambda_{k}^{\infty}, \lambda_{k}^{0}\right)$, all nontrivial solutions of (1.1) lie on two smooth curves $\Sigma_{k}^{ \pm}=\left\{\left(\lambda, u_{k}^{ \pm}(\lambda, \cdot)\right): \lambda \in\left(\lambda_{k}^{\infty}, \lambda_{k}^{0}\right)\right\}, \Sigma_{k}^{+}$and $\Sigma_{k}^{-}$join at $\left(\lambda_{k}^{0}, 0\right)$, and

$$
\lim _{\lambda \rightarrow\left(\lambda_{k}^{\infty}\right)^{+}}\left\|u_{k}^{ \pm}(\lambda, \cdot)\right\|_{L^{2}(\Omega)}=\infty
$$

(3) For a solution $(\lambda, u) \in \Sigma_{k}^{+} \cup \Sigma_{k}^{-}$, $u$ is nondegenerate and the Morse index $M(u)=k$.

We can also consider a similar semilinear problem:

$$
\begin{cases}\Delta u+\mu f(u)=0 & \text { in } \quad \Omega \\ u=0 & \text { on } \partial \Omega .\end{cases}
$$

The behavior of solution curves of (1.8) is the same as that of (1.1). (Notice the sign of $f(u)$ is positive in (1.8) instead of negative in (1.1).) In fact, if we define

$$
\mu_{k}^{0}=\frac{\lambda_{k}}{f^{\prime}(0)}, \mu_{k}^{\infty}=\frac{\lambda_{k}}{f^{\prime}(\infty)}, \mu_{k}^{m}=\frac{\lambda_{k}}{\inf _{u \in \mathbf{R}} f^{\prime}(u)}, \text { and } \mu_{k}^{M}=\frac{\lambda_{k}}{\sup _{u \in \mathbf{R}} f^{\prime}(u)},
$$

then

Theorem 1.3. Suppose that $f$ satisfies (f1), (f2) and (f3), and for $k=1,2, \cdots, N$, we assume that $\mu_{k}^{0} \leq \mu_{k+1}^{M}$. Then for (1.8), the conclusions in Theorem 1.2 hold with $\lambda$ 's replaced by $\mu$ 's. Similarly, if $f$ satisfies (f1), (f2) and (f4), and for $k=$ $1,2, \cdots, N$, we assume that $\mu_{k}^{m} \leq \mu_{k+1}^{0}$, then for (1.8), the conclusions in Theorem 1.1 hold with $\lambda$ 's replaced by $\mu$ 's.

The result that (1.8) has exactly two nontrivial solutions for $f$ satisfying (f1), (f2) and (f4) was first proved by Castro and Lazer [CL] using saddle-point reduction, and also by Ambrosetti and Mancini [AM] using Leray-Schauder degree theory. Our result is more general (does not require $f \in C^{2}$ ), our proof is different in using bifurcation technique, and we also consider the asymptotes of the solution curve.

The eigenvalue separation condition (1.5) is important for the exact multiplicity of the solutions. But for $n=1$ or the radial symmetric solutions on balls, even without the eigenvalue separation conditions, we can prove the same conclusions in Theorem 1.3 for $f$ satisfying (f4). In this case, we only consider (1.8), since that will be a generalization of earlier work by Chafee and Infante [CI]. We consider the semilinear elliptic equation (1.8) with $\Omega=B^{n}$, where $B^{n}$ is the unit ball in $\mathbf{R}^{n}$ for $n \geq 1$. The radially symmetric solutions to (1.8) satisfy

$$
u^{\prime \prime}+\frac{n-1}{r} u^{\prime}+\mu f(u)=0 \text { in }(0,1), u^{\prime}(0)=u(1)=0 .
$$




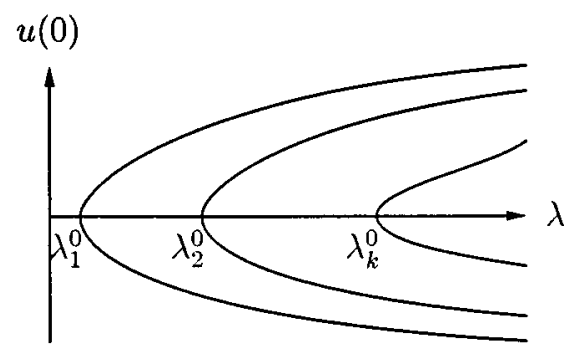

FIgURE 3. Bifurcation diagram for Theorem 1.4(1)

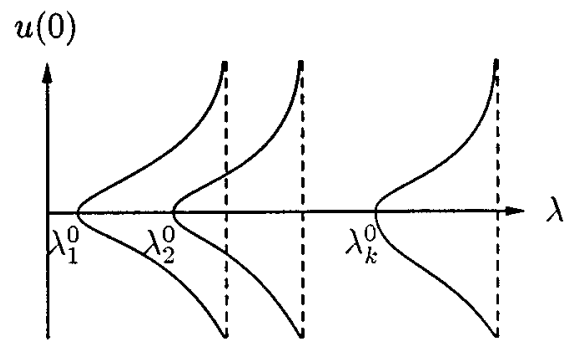

FiguRE 4. Bifurcation diagram for Theorem 1.4(2)

If $u$ is a solution to (1.10), we define the Radial Morse index $M_{r}(u)$ of $u$ to be the number of negative eigenvalues of the eigenvalue problem:

$$
w^{\prime \prime}+\frac{n-1}{r} w^{\prime}+\mu f^{\prime}(u) w=-\eta w \text { in }(0,1), w^{\prime}(0)=w(1)=0 .
$$

We have

Theorem 1.4. Suppose $f$ satisfies (f1), (f4) and $\lim _{|u| \rightarrow \infty} f(u) / u=f^{\prime}(\infty)$ exists $($ can be $-\infty)$. Let $\mu_{0}^{0}=0$, and $\mu_{k}^{0}(k>0)$ be the $k$-th eigenvalue of the eigenvalue problem:

$$
w^{\prime \prime}+\frac{n-1}{r} w^{\prime}+\mu f^{\prime}(0) w=0 \quad \text { in }(0,1), \quad w^{\prime}(0)=w(1)=0 .
$$

(1) If $f^{\prime}(\infty) \leq 0$, then (1.10) has exactly $2 k$ nontrivial solutions

$$
\left\{u_{i}^{+}(\mu, \cdot), u_{i}^{-}(\mu, \cdot): i=1,2, \cdots, k\right\}
$$

for $\lambda \in\left(\mu_{k}^{0}, \mu_{k+1}^{0}\right]$, where $k=0,1, \cdots$. Moreover, $u_{i}^{+}(\mu, 0)>0$ (resp. $\left.u_{i}^{-}(\mu, 0)<0\right)$, and $u_{i}^{+}(\mu, \cdot)$ (resp $\left.u_{i}^{-}(\mu, \cdot)\right)$ has exactly $i-1$ zeros in $(0,1)$. Furthermore, all solutions which have exactly $i-1$ zeros and satisfy $u(0)>0$ (or $u(0)<0)$ lie on a curve $\Sigma_{i}^{+}=\left\{\left(\mu, u_{i}^{+}(\mu, \cdot)\right): \mu>\mu_{i}^{0}\right\}$ (resp. $\Sigma_{i}^{-}=$ $\left.\left\{\left(\mu, u_{i}^{-}(\mu, \cdot)\right): \mu>\mu_{i}^{0}\right\}\right), M_{r}\left(u_{i}^{ \pm}\right)=i-1$, and there are no turning points on $\Sigma_{i}^{+}$(resp. $\left.\Sigma_{i}^{-}\right)$(see Figure 3).

(2) If $f^{\prime}(\infty)>0$, then a similar result holds, but $\Sigma_{i}^{ \pm}$exists only for $\mu \in\left(\mu_{i}^{0}, \mu_{i}^{\infty}\right)$, where $\mu_{i}^{\infty}$ is defined in (1.9) (see Figure 4). 
Theorem 1.1 is partially motivated by a recent paper [COW] by Castro, Ouyang and Wang, in which, the authors studied another semilinear boundary value problem:

$$
\begin{cases}\Delta u+\lambda u-h(x)|u|^{p-1} u=0 & \text { in } \Omega, \\ u=0 & \text { on } \partial \Omega,\end{cases}
$$

where $p>1$ and $h(x)$ is a nonnegative smooth function with $m(\{x \in \Omega: h(x)=$ $0\}) \neq 0$. Under eigenvalue separation conditions, they proved similar behavior of the first $N$ solution curves. Problem (1.13) was first studied by Ouyang [O].

For (1.1) and $f$ satisfying (f1) and (f3), it is well-known that the positive solution to (1.1) is unique for $\lambda>\lambda_{1}^{0}$; the same holds for equation (1.8) if $f$ satisfies (f1) and (f4). For $f$ satisfying (f4) in (1.1) (resp. (f3) in (1.8)), even for the positive solution branch, the eigenvalue separation condition is still needed. For (1.1) with (f4) and $\lambda_{1}+f^{\prime}(0) \leq \lambda_{2}+f^{\prime}(\infty)$, Hernández $[\mathrm{H}]$ proved the uniqueness of the positive solution for $\lambda \in\left(\lambda_{1}^{\infty}, \lambda_{1}^{0}\right)$, and for (1.8) with (f3), $f^{\prime \prime} \geq 0$, and $\lambda_{1} / f^{\prime}(0) \leq \lambda_{2} / f^{\prime}(\infty)$, the same result was proved by Amann [A2]. We should also mention that for Theorem 1.4, the special case of $n=1$ was proved by Chafee and Infante [CI] using phase portrait analysis.

We organize our paper in the following way. In Section 2, we give some preliminaries. In particular, some key comparison lemmas are proved. In Section 3, we prove Theorem 1.1 and we prove Theorem 1.4 in Section 4. The proofs of Theorems 1.2 and 1.3 are similar to that of Theorem 1.1, so we omit them. In the paper, we denote by $\|u\|_{2}$ the $L^{2}$ norm for $u \in L^{2}(\Omega)$, and by $m(\Omega)$ the Lebesgue measure of $\Omega$. Also $C$ stands for a generic positive constant.

\section{Preliminaries}

Let $W(x) \in L^{\infty}(\Omega)$. Consider the following eigenvalue problem:

$$
\begin{cases}\Delta \phi+W(x) \phi=-\mu_{i}(W) \phi & \text { in } \Omega, \\ \phi=0 & \text { on } \partial \Omega .\end{cases}
$$

It is well-known that, for $i=1,2, \cdots$,

$$
\mu_{i}(W)=\operatorname{Min}_{i} \operatorname{Max}_{i} \frac{\int_{\Omega}\left(|\nabla z|^{2}-W(x) z^{2}\right) d x}{\int_{\Omega} z^{2} d x}
$$

where $M a x_{i}$ is over all $z(\neq 0) \in T_{i}$, and $\operatorname{Min}_{i}$ is over all $i$-dimensinal subspaces $T_{i}$ of $H_{0}^{1}(\Omega)$.

Lemma 2.1. Suppose that $W_{1}, W_{2} \in L^{\infty}(\Omega)$ satisfy $W_{2}(x) \geq W_{1}(x)$ a.e.; then $\mu_{i}\left(W_{2}\right) \leq \mu_{i}\left(W_{1}\right)$. If in addition $m\left(\left\{W_{2}>W_{1}\right\}\right)>0$, then $\mu_{i}\left(W_{2}\right)<\mu_{i}\left(W_{1}\right)$.

Proof. From the variational characterization (2.2), we see immediately that $\mu_{i}\left(W_{2}\right)$ $\leq \mu_{i}\left(W_{1}\right)$. Suppose that $m\left(\left\{W_{2}>W_{1}\right\}\right)>0$. For $i \geq 1$, let $\psi_{j}\left(W_{1}\right)$ be the eigenfunctions corresponding to $\mu_{j}\left(W_{1}\right), j=1,2, \cdots, i$, and $M=\operatorname{span}\left\{\psi_{j}\left(W_{1}\right)\right.$ : $1 \leq j \leq i\}$. Then

$$
\begin{aligned}
\mu_{i}\left(W_{1}\right) & =\max _{z \in M \backslash\{0\}} \frac{\int_{\Omega}\left(|\nabla z|^{2}-W_{1}(x) z^{2}\right) d x}{\int_{\Omega} z^{2} d x} \\
& >\max _{z \in M \backslash\{0\}} \frac{\int_{\Omega}\left(|\nabla z|^{2}-W_{2}(x) z^{2}\right) d x}{\int_{\Omega} z^{2} d x} \geq \mu_{i}\left(W_{2}\right) .
\end{aligned}
$$


We can achieve strict inequality in the above because, by the unique continuation property (see [DG]), $m(\{x \in \Omega: z(x)=0\})=0$ for any nonzero $z \in M$, thus $\int_{\Omega} W_{1}(x) z^{2} d x<\int_{\Omega} W_{2}(x) z^{2} d x$.

Lemma 2.2. Suppose that $f$ satisfies (f1), (f2) and (f3), and $u$ is a nontrivial solution of (1.1). Then

$$
\begin{gathered}
\mu_{j}\left(\lambda-f^{\prime}(0)\right)<\mu_{j}(\lambda-f(u) / u)<\mu_{j}\left(\lambda-f^{\prime}(u)\right)<\mu_{j}\left(\lambda-\sup _{u \in \mathbf{R}} f^{\prime}(u)\right), \\
\mu_{j}(\lambda-f(u) / u)<\mu_{j}\left(\lambda-f^{\prime}(\infty)\right) .
\end{gathered}
$$

Proof. (2.4) can be obtained by Lemma 2.1 by observing that (f2) and (f3) imply

$$
f^{\prime}(0) \leq \frac{f(u(x))}{u(x)} \leq f^{\prime}(u(x)) \leq \sup _{u \in \mathbf{R}} f^{\prime}(u), \text { and } \frac{f(u(x))}{u(x)} \leq f^{\prime}(\infty) \quad \text { a.e. }
$$

Note that again by the unique continuation property, $m(\{x \in \Omega: u(x)=0\})=$ 0 .

We define $I_{j}=\left(\lambda_{j}^{0}, \lambda_{j}^{\infty}\right), \widetilde{I}_{j}=\left(\lambda_{j}^{0}, \lambda_{j}^{M}\right)$.

Lemma 2.3. Suppose that $f$ satisfies (f1), (f2) and (f3).

(1) If $\lambda \notin \bigcup_{j \geq 1} I_{j}$, then (1.1) has no nontrivial solution.

(2) If $\lambda \in I_{j} \subset \widetilde{I}_{j}, \lambda \notin \widetilde{I}_{k}$ for any other $k \neq j$, and $u$ is a nontrivial solution to (1.1), then $M(u)=j-1$ and $\mu_{j}\left(\lambda-f^{\prime}(u)\right)>0$.

Proof. (1) If $u$ is a nontrivial solution of (1.1), then 0 is an eigenvalue of (2.1) with $W(x)=\lambda-f(u(x)) / u(x)$, i.e., $0=\mu_{j}(\lambda-f(u) / u)$ for some $j \geq 1$. By (2.4), $\mu_{j}\left(\lambda-f^{\prime}(0)\right)<\mu_{j}(\lambda-f(u) / u)=0<\mu_{j}\left(\lambda-f^{\prime}(\infty)\right)$, thus $\lambda \in I_{j}$.

(2) If $\lambda \in I_{j}$, and $\lambda \notin \widetilde{I}_{k}$ for any other $k \neq j$, then for any $k<j, \mu_{k}\left(\lambda-f^{\prime}(0)\right)$ and $\mu_{k}\left(\lambda-\sup _{u \in \mathbf{R}} f^{\prime}(u)\right)$ are both negative, and by $(2.4), \mu_{k}\left(\lambda-f^{\prime}(u)\right)<0$ and $\mu_{k}(\lambda-f(u) / u)<0$. Similarly, for $k>j, \mu_{k}\left(\lambda-f^{\prime}(u)\right)>0$ and $\mu_{k}(\lambda-f(u) / u)>0$. On the other hand, $0=\mu_{l}(\lambda-f(u) / u)$ for some $l \geq 1$. Hence $l=j$, and by Lemma $2.2 \mu_{j}\left(\lambda-f^{\prime}(u)\right)>0$. Therefore $M(u)=j-1$ and $\mu_{j}\left(\lambda-f^{\prime}(u)\right)>0$.

Corollary 2.4. Suppose that $f$ satisfies (f1), (f2), (f3) and (1.5). If $\lambda \in\left[\lambda_{j}^{\infty}, \lambda_{j+1}^{0}\right]$, then (1.1) has only the trivial solution. If $\lambda \in\left(\lambda_{j}^{0}, \lambda_{j}^{\infty}\right)$ and $u$ is a nontrivial solution of (1.1), then $u$ is nondegenerete and $M(u)=j-1$.

Next we need to identify where the solution curves of (1.1) will blow up. We say that $\lambda_{*}$ is a point where a bifurcation from infinity occurs for (1.1) if there exists a sequence $\lambda^{k} \rightarrow \lambda_{*}$ as $k \rightarrow \infty$ such that there is a solution $u_{k}$ of (1.1) with $\lambda=\lambda^{k}$ and $\left\|u_{k}\right\|_{2} \rightarrow \infty$.

Lemma 2.5. Suppose that $f$ satisfies (f1) and (f2), and $\lambda_{*}$ is a point where bifurcation from infinity occurs for (1.1). Then $\lambda_{*}-f^{\prime}(\infty)=\lambda_{k}$ for some $k \in \mathbf{N}$.

Proof. We define $\phi_{k}(x)=\left\|u_{k}\right\|_{2}^{-1} u_{k}(x)$. Then $\phi_{k}$ satisfies

$$
\Delta \phi_{k}+\lambda^{k} \phi_{k}-\frac{f\left(u_{k}\right)}{u_{k}} \phi_{k}=0 .
$$

We multiply (2.5) by $\phi_{k}$ and integrate over $\Omega$ to obtain

$$
\int_{\Omega}\left|\nabla \phi_{k}\right|^{2} d x+\int_{\Omega} \frac{f\left(u_{k}\right)}{u_{k}} \phi_{k}^{2} d x=\lambda^{k} \int_{\Omega} \phi_{k}^{2} d x=\lambda^{k} \leq C .
$$


Since $f(u) / u$ is bounded, then there exists $\phi \in H_{0}^{1}(\Omega)$ such that $\phi_{k}$ has a subsequence (which we still denote by $\left\{\phi_{k}\right\}$ ) converging to $\phi$ strongly in $L^{2}(\Omega)$, and weakly in $H_{0}^{1}(\Omega)$. Let $\Omega^{+}=\{x \in \Omega: \phi(x)>0\}$ and $\Omega^{-}=\{x \in \Omega: \phi(x)<0\}$. Then $u_{k}(x)=\left\|u_{k}\right\|_{2} \phi_{k}(x) \rightarrow \pm \infty$ as $k \rightarrow \infty$ for $x \in \Omega^{+} \cup \Omega^{-}$, thus

$$
\frac{f\left(u_{k}(x)\right)}{u_{k}(x)} \rightarrow f^{\prime}(\infty), \quad x \in \Omega^{+} \cup \Omega^{-} .
$$

Let $\psi \in C_{0}^{1}(\Omega)$. We multiply (2.5) by $\psi$ and integrate over $\Omega$ to obtain

$$
\int_{\Omega} \nabla \phi_{k} \cdot \nabla \psi d x+\int_{\Omega} \frac{f\left(u_{k}\right)}{u_{k}} \phi_{k} \psi d x-\lambda^{k} \int_{\Omega} \phi_{k} \psi d x=0 .
$$

By the weak convergence of $\phi_{k}$ and (2.7), we conclude that $\phi$ is a weak solution of

$$
\begin{cases}\Delta \phi+\left[\lambda_{*}-f^{\prime}(\infty)\right] \phi=0 & \text { in } \Omega, \\ \phi=0 & \text { on } \partial \Omega .\end{cases}
$$

Therefore, $\lambda_{*}-f^{\prime}(\infty)=\lambda_{k}$ for some $k$.

At last, let $(\lambda(s), u(s))$ with $|s| \leq \delta$ be the solution curve of (1.1) bifurcating from the trivial solutions at a simple eigenvalue $\lambda_{*}=\lambda_{j}^{0}$ (see Theorem 1.7 in [CR1]). We need to know if $\lambda(s) \geq \lambda *$ or $\lambda(s) \leq \lambda *$.

Lemma 2.6. Suppose that $f$ satisfies (f1) and

$(\mathfrak{f} 3)^{\prime} f(u) / u$ is increasing in $\left(0, \delta_{1}\right)$ and is decreasing in $\left(-\delta_{1}, 0\right)$ for some $\delta_{1}>0$. If $\lambda_{*}=\lambda_{j}^{0}$ is a point where a bifurcation from the trivial solutions occurs for (1.1), then $\lambda(s)>\lambda *$ for $0<|s|<\delta$.

Proof. Let $\mu_{k}(s)=\mu_{k}\left(\lambda(s)-f^{\prime}(u(s))\right)$ and $\overline{\mu_{k}}(s)=\mu_{k}(\lambda(s)-f(u(s)) / u(s))$ for $k \in \mathbf{N}$. Then by Lemma $2.2, \mu_{k}(s)>\overline{\mu_{k}}(s)$ for $|s|$ sufficiently small. On the other hand, $0=\overline{\mu_{k}}(s)$ for some $k$. Since $\overline{\mu_{j}}(0)=0$ and $\overline{\mu_{k}}(s)$ is continuous with respect to $s$, then $\overline{\mu_{j}}(s)=0$ for $0<|s|<\delta$. Thus $\mu_{j}(s)>0$ and $M(u(s))=j-1$. Now we can apply Theorem 1.16 in [CR2], and obtain $\operatorname{sign}\left(\lambda^{\prime}(s)\right)=\operatorname{sign}(s)$ for $0<|s|<\delta$. In particular, $\lambda(s)>\lambda *$ for $0<|s|<\delta$.

\section{Proof of Theorem 1.1}

Proof of Theorem 1.1. By Theorem 1.7 in [CR1], for $1 \leq j \leq N,\left(\lambda_{j}^{0}, 0\right)$ is a bifurcation point (recall that $\lambda_{j}$ is simple). Let $w$ be a solution of the linearized equation (1.4) with $u=0$ and $\lambda=\lambda_{j}^{0}$, and let $Z$ be any complement of $\operatorname{span}\{w\}$ in $L^{2}(\Omega)$. Then there exists $\delta>0$, and continuously differentiable functions $\lambda:(-\delta, \delta) \rightarrow \mathbf{R}$ and $\psi:(-\delta, \delta) \rightarrow Z$ such that $\lambda(0)=\lambda_{j}^{0}, \psi(0)=0$, and if $u(s)=s w+s \psi(s)$ for $s \in(-\delta, \delta)$, then $(\lambda(s), u(s))$ is a solution to (1.1). Moreover, all the solutions to (1.1) in a neighborhood of $\left(\lambda_{j}^{0}, 0\right)$ consist precisely of the curves $u=0$ and $(\lambda(s), u(s)), s \in(-\delta, \delta)$. By Lemma 2.6, $\lambda(s)>\lambda_{j}^{0}$ for $s \in(-\delta, \delta)$. Therefore, there exists $\delta_{1}>0$ such that for $0<\lambda-\lambda_{j}^{0} \leq \delta_{1}$, (1.1) has exactly two nontrivial solutions near 0 . We denote by $u_{j}^{+}(\lambda, \cdot)$ the solution with $\lambda=\lambda(s)$, $s>0$, and denote by $u_{j}^{-}(\lambda, \cdot)$ the solution with $\lambda=\lambda(s), s<0$. We also define $\Sigma_{j}^{ \pm}=\left\{\left(\lambda, u_{j}^{ \pm}(\lambda, \cdot)\right): \lambda \in\left(\lambda_{j}^{0}, \lambda_{j}^{0}+\delta_{1}\right]\right\}$.

By Corollary 2.4, $u_{j}^{ \pm}(\lambda, \cdot)$ are nondegenerate, thus by the Implicit Function Theorem, $\Sigma_{j}^{ \pm}$can be extended further beyond $\lambda=\lambda_{j}^{0}+\delta_{1}$. Then we can continue $\Sigma_{j}^{ \pm}$ in the $\lambda$ direction to the right. Let $\lambda^{*}=\sup \left\{\lambda>\lambda_{j}^{0}+\delta_{1}:\left(\lambda, u_{j}^{+}(\lambda, \cdot)\right)\right.$ exists $\}$. 
By Corollary 2.4, $\lambda^{*} \leq \lambda_{j}^{\infty}$. If $\underline{\lim }_{\lambda \rightarrow\left(\lambda^{*}\right)^{-}}\left\|u_{j}^{+}(\lambda, \cdot)\right\|_{2}<\infty$, then there exist $K>0$ and a sequence $\left\{\lambda^{k}\right\}$ such that $\left\|u_{j}^{+}\left(\lambda^{k}, \cdot\right)\right\|_{2} \rightarrow K$. From equation (1.1), $\left\|u_{j}^{+}\left(\lambda^{k}, \cdot\right)\right\|_{H_{0}^{1}(\Omega)} \leq C\left\|u_{j}^{+}\left(\lambda^{k}, \cdot\right)\right\|_{2} \leq C K$. Therefore, there exists $v \in H_{0}^{1}(\Omega)$ such that $u_{j}^{+}\left(\lambda^{k}, \cdot\right)$ has a subsequence converging to $v$ strongly in $L^{2}(\Omega)$, and weakly in $H_{0}^{1}(\Omega)$. Moreover, $\|v\|_{2}=K$. If $K>0$, then $v$ is a weak solution to (1.1), with $\lambda=\lambda^{*}$. By the standard regularity theory for elliptic equations, $v$ is a classical solution, and nondegenerate by Corollary 2.4 , so we can extend $\Sigma_{j}^{+}$further beyond $\lambda=\lambda^{*}$, which is a contradiction to the definition of $\lambda^{*}$. If $K=0$, then $\lambda^{*}$ is a point where a bifurcation from the zero solutions occurs. But $\lambda_{j}^{0}<\lambda^{*} \leq \lambda_{j}^{\infty} \leq \lambda_{j+1}^{0}$, so that is impossible. Therefore $\lim _{\lambda \rightarrow\left(\lambda^{*}\right)^{-}}\left\|u_{j}^{+}(\lambda, \cdot)\right\|_{2}=\infty$. By Lemma 2.5 and $\lambda^{*} \leq \lambda_{j}^{\infty}, \lambda^{*}=\lambda_{j}^{\infty}$. By Corollary 2.4, $M(u)=j-1$ for any solution $u$ on $\Sigma_{j}^{+}$.

All this can also be done for $\Sigma_{j}^{-}$. Therefore, $\Sigma_{j}^{ \pm}$are solution curves which can be parameterized by $\lambda$ with $\lambda \in\left(\lambda_{j}^{0}, \lambda_{j}^{\infty}\right)$.

Finally we exclude the possibility of solutions with $\lambda \in I_{j}$ which are not on $\Sigma_{j}^{ \pm}$. Suppose such a solution $u(\lambda)$ exists for some $\lambda \in I_{j}$. Then again, $u(\lambda)$ is nondegenerate, so we can extend $u(\lambda)$ to form a curve (which we still denote by $u(\lambda)$ ). Let $\lambda_{*}=\sup \{\lambda: u(\lambda)$ is a nontrivial solution to (1.1)\}. By Corollary 2.4, $\lambda_{*} \leq \lambda_{\infty}^{j}$. Then as in the last paragraph, we can prove that $\lim _{\lambda \rightarrow\left(\lambda_{*}\right)}\|u(\lambda)\|_{2}=$ $\infty$, then $\lambda_{*}=\lambda_{j}^{\infty}$. But all solutions near $\left(\lambda_{j}^{\infty}, \infty\right)$ can be parameterized by $s$ for $|s|>\delta$, thus all these solutions are on $\Sigma_{k}^{ \pm}$. Therefore, there is no other solution to (1.1) for $\lambda \in I_{j}$ besides those on $\Sigma_{j}^{ \pm}$. This completes the proof.

\section{Proof of Theorem 1.4}

Bifurcations from simple eigenvalues still occur for (1.10). But for this case, a stronger result holds, i.e. we also know about the nodal structure of the solutions to (1.10). It is well-known that (1.12) possesses a sequence of eigenvalues $\left\{\mu_{j}\right\}$ such that $\mu_{1}<\mu_{2}<\cdots<\mu_{j} \rightarrow \infty$ as $j \rightarrow \infty, \mu_{j}$ is simple, any eigenfunction $\phi_{j}$ corresponding to $\mu_{j}$ has exactly $j-1$ zeros in $(0,1)$ and all zeros of $\phi_{j}$ in $[0,1]$ are simple. (A simple zero of $\phi_{j}$ is a point $r \in[0,1]$ such that $\phi_{j}(r)=0$ and $\phi_{j}^{\prime}(r) \neq 0$.) We define

$$
\begin{aligned}
S_{j}^{+}= & \left\{v: v^{\prime}(0)=v(1)=0, v(0)>0, v \text { has exactly } j-1\right. \text { zeros in } \\
& (0,1), \text { and all zeros of } v \text { in }[0,1] \text { are simple }\}, \\
S_{j}^{-}= & -S_{j}^{+}, \text {and } S_{j}=S_{j}^{+} \cup S_{j}^{-} .
\end{aligned}
$$

Then Theorem 2.3 of [R1] can be applied to (1.10), and we have the following lemma:

Lemma 4.1. For any $k \in \mathbf{N}$, (1.10) possesses a component of solutions $\Sigma_{k}$ in $\mathbf{R} \times E$ with $\Sigma_{k} \subset\left(\mathbf{R} \times S_{k}\right) \cup\left\{\left(\mu_{k}^{0}, 0\right)\right\}$ and $\Sigma_{k}$ is unbounded, where $E=C^{1}[0,1]$.

On the other hand, the solution set of (1.10) can be parameterized by $u(0)$ in the following sense (a proof can be found in Castro, Gadam and Shivaji [CGS]):

Lemma 4.2. Given $j \in \mathbf{N}$, for each $d \neq 0$ there exists at most one $\mu>0$ such that (1.10) has a solution $u(\mu, r)$ which has exactly $j-1$ zeros in $(0,1)$ and satisfies $u(\mu, 0)=d$. 
We prove Theorem 1.4 in a sequence of lemmas. Suppose that $u(\mu, r)$ is a solution of $(1.10)$, and we define $w(\mu, r)$ to be the solution of

$$
w^{\prime \prime}+\frac{n-1}{r} w^{\prime}+\mu f^{\prime}(u(\mu, r)) w=0 \text { in }(0,1), \quad w^{\prime}(0)=0, \quad w(0)=1 .
$$

Lemma 4.3. Suppose that $u(\mu, r)$ is a solution of $(1.10)$, and $w(\mu, r)$ is the solution of (4.1). Then $M(u(\mu, \cdot))=k$ if and only if $w(\mu, r)$ has exactly $k$ zeros in $(0,1)$.

Proof. Let $\varphi_{i}$ be the eigenfunction of (1.11) corresponding to eigenvalue $\eta_{i}$ for $i \geq 1$. Then $w(r)=w(\mu, r)$ and $\varphi_{i}(r)$ satisfy the following equations respectively:

$$
\begin{aligned}
& \left(r^{n-1} w^{\prime}\right)^{\prime}+\mu r^{n-1} f^{\prime}(u) w=0, \\
& \left(r^{n-1} \varphi_{i}^{\prime}\right)^{\prime}+\mu r^{n-1} f^{\prime}(u) \varphi_{i}=-\eta_{i} r^{n-1} \varphi_{i} .
\end{aligned}
$$

We notice that $M(u(\mu, \cdot))=k$ if and only if then $\eta_{k}<0$ and $\eta_{k+1} \geq 0$. We extend $\varphi_{k}, \varphi_{k+1}$ and $w$ to $(-1,0)$ evenly; then $\varphi_{k}$ has $2 k$ zeros in $[-1,1]$ and $\varphi_{k+1}$ has $2 k+2$ zeros. Therefore, by the Sturm comparison lemma, $M(u(\mu, \cdot))=k$ if and only if $w$ has exactly $k$ zeros in $(0,1)$.

Lemma 4.4. Under the hypothesis of Theorem 1.4, if $(\mu, u(\mu, \cdot)) \in \Sigma_{k} \backslash\left\{\left(\mu_{k}^{0}, 0\right)\right\}$, then $M(u(\mu, \cdot))=k-1$, and $w(\mu, 1) \neq 0$.

Proof. Since $u \in S_{k}$, then all zeros of $u$ are simple. By the conditions in Theorem 1.4 and the maximal principle, $u$ has no positive local minimum and negative local maximum. Therefore there exists

$$
0=a_{1}<b_{1}<a_{2}<b_{2}<\cdots<a_{k-1}<b_{k-1}<a_{k}<b_{k}=1,
$$

such that $u^{\prime}\left(a_{i}\right)=0, u\left(b_{i}\right)=0, i=1,2, \cdots, k$. The functions $u(r), w(r)=w(\mu, r)$ and $u_{r}(r)$ satisfy the following equations respectively:

$$
\begin{aligned}
& \left(r^{n-1} u^{\prime}\right)^{\prime}+\mu r^{n-1}[f(u) / u] u=0, \\
& \left(r^{n-1} w^{\prime}\right)^{\prime}+\mu r^{n-1} f^{\prime}(u) w=0, \\
& \left(r^{n-1} u_{r}^{\prime}\right)^{\prime}+\left[\mu r^{n-1} f^{\prime}(u)-\mu(n-1) r^{n-3}\right] u_{r}=0 .
\end{aligned}
$$

Since $f$ satisfies (f4), then for any $r \in(0,1)$,

$$
\frac{f(u)}{u} \geq f^{\prime}(u) \geq f^{\prime}(u)-\frac{n-1}{r^{2}} .
$$

Then by the Sturm comparison lemma, between any two consecutive zeros of $u_{r}$, there exists at least one zero of $w$. Thus $w$ has at least one zero in $\left(a_{i}, a_{i+1}\right)$ for $i=1,2, \cdots, k-1$ and $w$ has at least $k-1$ zeros in $(0,1)$. On the other hand, still by the Sturm comparison lemma, between any two consecutive zeros of $w$, there exists at least one zero of $u$. Suppose that $w$ has at least $k$ zeros in $(0,1)$. We can extend $u$ and $w$ to $(-1,0)$ evenly. Then $w$ has at least $2 k$ zeros in $(-1,1)$, and all zeros of $w$ are also simple by the uniqueness of ordinary differential equations. Hence $u$ has at least $2 k-1$ zeros in $(-1,1)$, which contradicts that $u \in S_{k}$ and has only $2 k-2$ zeros in $(-1,1)$. Therefore $w$ has exactly $k-1$ zeros in $(0,1)$, and by Lemma $4.3, M(u(\lambda, \cdot))=k-1$. The proof above also implies that the $k-1$ zeros of $w$ must be in an alternating order with the zeros of $u$. In particular, there exists $r_{0} \in\left(b_{k-1}, 0\right)$ such that $w\left(r_{0}\right)=0$. Suppose that $w(1)=0$; then there exists another zero of $u$ in $\left(r_{0}, 1\right)$, which is a contradiction. Therefore, $w(1) \neq 0$. 
Proof of Theorem 1.4. By Lemma 4.1, for $k \in \mathbf{N}$, there exists a solution curve $\Sigma_{k}$ bifurcating from $\left(\mu_{k}^{0}, 0\right)$, and the solution $u(\mu, \cdot)$ on $\Sigma_{k}$ has exactly $k-1$ zeros in $(0,1)$. By Lemma 4.4, $M(u(\mu, \cdot))=k-1$; then the $k$-th eigenvalue $\eta_{k}[u(\mu, \cdot)]>0$. Therefore, by Lemma 2.5, the solution curve $\Sigma_{k}$ bends to the right of $\mu_{k}^{0}$. We denote the upper branch $(u(0)>0)$ of $\Sigma_{k}$ by $\Sigma_{k}^{+}$, and the lower branch $(u(0)<0)$ by $\Sigma_{k}^{-}$. By Lemma 4.2 , we can parameterize $\Sigma_{k}$ by $s=u(0)$, so letting $\Sigma_{k}=(\mu(s), u(s))$, we can continue $\Sigma_{k}^{+}$to the right as long as $\mu^{\prime}(s)>0$. But by Lemma 4.4, 0 cannot be an eigenvalue of (1.11) for any solution $u(\mu, \cdot)$ on $\Sigma_{k}^{+}$. Then $\Sigma_{k}^{+}$can be extended to $\mu_{\infty}=\sup \left\{\mu:(\mu, u(\mu, \cdot)) \in \Sigma_{k}^{+}\right\}$with $\mu$ monotone increasing. If $f^{\prime}(\infty) \leq 0$, we claim $\mu_{\infty}=\infty$. In fact, it is well-known that for such $f,(1.1)$ has a positive solution and a negative solution for all $\mu>\mu_{1}^{0}$ (see [A1]), so $\Sigma_{1}$ can be extended to $\mu=\infty$. By Lemma 4.2, $\Sigma_{k}$ can be regarded as curves on $\mathbf{R}^{+} \times \mathbf{R}=\{(\mu, u(0))\}$. Since $\Sigma_{k}$ is unbounded and $\Sigma_{k} \cap \Sigma_{1}=\emptyset$ for $k>1$, then $\Sigma_{k}$ can also be extended to $\mu=\infty$. If $f^{\prime}(\infty)>0$, we claim that $\mu_{\infty}=\mu_{k}^{\infty}$. In fact, by the shooting method, we can prove that for any $d \neq 0,(1.1)$ has a solution $u$ with exactly $k-1$ zeros in $(0,1)$ for $k \geq 1$. Thus by Lemma $4.2, \Sigma_{k}^{+}$can be regarded as the graph of a function $d \mapsto \mu(d)$ for $d \in(0, \infty)$. But by the result of [R2], $\lim _{d \rightarrow \infty} \mu(d)=\mu_{k}^{\infty}$, and by Lemma 4.4, $\mu(d)$ has no critical point, thus $\mu^{\prime}(d)>0$. Therefore $\mu_{\infty}=\mu_{k}^{\infty}$.

\section{Concluding Remark}

To conclude our paper, we have a remark on the validity of the eigenvalue separation condition (1.5). (Similar analysis is also true for other eigenvalue separation conditions.) We note that (1.5) is equivalent to $\sup _{u \in \mathbf{R}} f^{\prime}(u)-f^{\prime}(0) \leq \lambda_{j+1}-\lambda_{j}$ for $j=1,2, \cdots, N$. By a formula due to Weyl, we have $\lambda_{j} \sim(j / m(\Omega))^{2 / n}$, where $n$ is the spatial dimension. Therefore, when $n=1, \lambda_{j+1}-\lambda_{j} \rightarrow \infty$ as $j \rightarrow \infty$, then $\sup _{u \in \mathbf{R}} f^{\prime}(u)-f^{\prime}(0) \leq \lambda_{j+1}-\lambda_{j}$ is true for all $j$ large enough. But Theorem 1.3 is an even stronger result for $n=1$. For $n=2, \lambda_{j+1}-\lambda_{j} \rightarrow C>0$ as $j \rightarrow \infty$. Therefore, if we assume $\sup _{u \in \mathbf{R}} f^{\prime}(u)-f^{\prime}(0) \leq \inf _{j \in \mathbf{N}}\left(\lambda_{j+1}-\lambda_{j}\right)$ for all $j$, then by the proof of Theorem 1.1, we can prove not only the first $N$ but all solution curves are monotone, which is stronger than the conclusion of Theorem 1.1. For $n \geq 3$, $\lambda_{j+1}-\lambda_{j} \rightarrow 0$, then the $\lambda$ range of solution curves must have overlaps for large $j$.

\section{ACKNOWLEDGEMENT}

The authors wish to thank Professor Peter W. Bates for the careful reading and helpful suggestions of an earlier version of this paper, and thank the referee for some helpful comments and suggestions.

\section{REFERENCES}

[A1] Amann, Herbert, Fixed point equations and nonlinear eigenvalue problems in ordered Banach space. SIAM Review 18 (1976), 620-709. MR 54:3519

[A2] Amann, Herbert, Nonlinear eigenvalue problems having precisely two solutions. Math. Z. 150 (1976), no. 1, 27-37. MR 54:721

[AM] Ambrosetti, Antonio; Mancini, Giovanni, Sharp nonuniqueness results for some nonlinear problems. Nonlinear Anal. 3 (1979), no. 5, 635-645. MR 80k:47073

[CGS] Castro, Alfonso; Gadam, S.; Shivaji, R., Branches of radial solutions for semipositone problems. J. Diff. Equations 120 (1995), 30-45. MR 96h:35058

[CL] Castro, Alfonso; Lazer, A. C., Critical point theory and the number of solutions of a nonlinear Dirichlet problem. Ann. Mat. Pura Appl. (4) 120 (1979), 113-137. MR 81d:58022 
[CI] Chafee, N.; Infante, E. F., A bifurcation problem for a nonlinear partial differential equation of parabolic type. Applicable Anal. 4 (1974/75), 17-37. MR 55:13084

[COW] Castro, Alfonso; Ouyang, Tiancheng; Wang, Junping, Bifurcation from a simple eigenvalue in a superlinear boundary value problem (preprint) (1997).

[CR1] Crandall, Michael G.; Rabinowitz, Paul H, Bifurcation from simple eigenvalues. J. Functional Analysis 8 (1971), 321-340. MR 44:5836

[CR2] Crandall, Michael G.; Rabinowitz, Paul H. Bifurcation, perturbation of simple eigenvalues and linearized stability. Arch. Rational Mech. Anal. 52 (1973), 161-180. MR 49:5962

[DG] de Figueiredo, Djairo G.; Gossez, Jean-Pierre, Strict monotonicity of eigenvalues and unique continuation. Comm. Partial Differential Equations 17 (1992), no. 1-2, 339-346. MR 93b:35098

[H] Hernández, Jesús, Qualitative methods for nonlinear diffusion equations. Nonlinear diffusion problems (Montecatini Terme, 1985), 47-118, Lecture Notes in Math., 1224, Springer, Berlin-New York, 1986. MR 88b:35076

[O] Ouyang, Tiancheng, On the positive solutions of semilinear equations $\Delta u+\lambda u-h u^{p}=0$ on the compact manifolds. Trans. Amer. Math. Soc. 331 (1992), no. 2, 503-527. MR 92h:35012

[R1] Rabinowitz, Paul H., Some global results for nonlinear eigenvalue problems. J. Func. Anal. 7 (1971), 487-513. MR 46:745

[R2] Rabinowitz, Paul H., On bifurcation from infinity. J. Diff. Equations 14 (1973), 462-475. MR 48:7047

Department of Mathematics, Tulane University, New Orleans, Louisiana 70118

E-mail address: shij@math.tulane.edu

Department of Mathematics, Brigham Young University, Provo, Utah 84602-6539

E-mail address: junw@math.byu.edu 